\title{
Design of a Model Reference Adaptive Controller for an Unmanned Air Vehicle*
}

\author{
Luis G. Crespo ${ }^{\dagger}$ \\ National Institute of Aerospace, Hampton, VA, 23666, USA \\ Megumi Matsutani ${ }^{\ddagger}$, and Anuradha M. Annaswamy $\S$ \\ Massachusetts Institute of Technology, Cambridge, MA, 02139, USA
}

\begin{abstract}
This paper presents the "Adaptive Control Technology for Safe Flight (ACTS)" architecture, which consists of a non-adaptive controller that provides satisfactory performance under nominal flying conditions, and an adaptive controller that provides robustness under off nominal ones. The design and implementation procedures of both controllers are presented. The aim of these procedures, which encompass both theoretical and practical considerations, is to develop a controller suitable for flight. The ACTS architecture is applied to the Generic Transport Model developed by NASA-Langley Research Center. The GTM is a dynamically scaled test model of a transport aircraft for which a flight-test article and a high-fidelity simulation are available. The nominal controller at the core of the ACTS architecture has a multivariable LQR-PI structure while the adaptive one has a direct, model reference structure. The main control surfaces as well as the throttles are used as control inputs. The inclusion of the latter alleviates the pilot's workload by eliminating the need for cancelling the pitch coupling generated by changes in thrust. Furthermore, the independent usage of the throttles by the adaptive controller enables their use for attitude control. Advantages and potential drawbacks of adaptation are demonstrated by performing high fidelity simulations of a flight-validated controller and of its adaptive augmentation.
\end{abstract}

Keywords: Adaptive control, engine control, safety, UAV.

\section{Introduction}

An adaptive reconfigurable controller autonomously changes the controller gains to maintain satisfactory performance when unforeseen changes in the system dynamics occur. Adaptive control has the potential to improve flight safety, as loss-of-control is one of the major causes of abnormal flight and fatal accidents. Over the past three decades, adaptive control has been developed extensively and its main performance and robustness properties have been established $[1,5,6,9,10]$.

In this paper we design a controller for the Generic Transport Model (GTM). The GTM is a dynamically scaled test model of a transport aircraft for which NASA Langley has developed a flight-test article and a high-fidelity simulation. This simulation uses non-linear aerodynamic models extracted from wind tunnel and system identification data, and considers avionics, sensor dynamics, engine dynamics, atmospheric conditions, sensor noise and biases, telemetry effects, etc. Overall, the open-loop plant has 278 states. Since this model, as the vehicle dynamics itself, departs considerably from the Linear Time Invariant (LTI) setting supporting the theory, it remains to be determined if the improvements in stability, safety, and performance caused by adaptation are realized in practice.

The nominal controller architecture consists of (i) a longitudinal multivariable controller having the elevator and the throttle inputs to both engines as control inputs and (ii) a lateral/directional multivariable controller having the ailerons and rudders as inputs. A fixed control allocation of this controller's output precludes using the engines for attitude control. On the other hand, the adaptive controller manipulates each of the five control inputs independently, therefore, it is solely responsible for generating differential thrust. Because of the placement of the engines and the orientation of the thrust vector relative to the body axes, changes in thrust create a pitching moment disturbance that

\footnotetext{
*This work was supported by the NRA NNX08AC62A of the IRAC project of NASA.

$\dagger$ Senior Research Scientist, 100 Exploration Way, AIAA Member.

¥Graduate Student, Mechanical Engineering, 77 Massachusetts Avenue, Room 3-441.

${ }^{\S}$ Senior Research Scientist, Mechanical Engineering, 77 Massachusetts Avenue, Room 3-339A.
} 
must be cancelled by the elevators. Auto throttle designs that only depend on the aircraft velocity rely on the pilot's ability to generate a suitable set of pitch commands to attain the desired cancellation. The ACTS controller pursues this cancellation automatically, thereby considerably reducing the pilot's workload.

Simulation studies are used to illustrate some advantages and liabilities of adaptation. While these simulations only give a local notion of the performance and robustness characteristics of both the nominal and adaptive controllers, the companion paper [3] evaluates these characteristics from a more global perspective [2,4]. Developments that enable tuning the control parameters to improve the resulting system's robustness are also presented.

This paper is organized as follows. Section $\Pi$ presents the control structure of both the baseline and adaptive controllers. This is followed by Section III where a departure in the controller's implementation from the LTI framework supporting the adaptive control design procedure is made. Section IV demonstrates some advantages and potential drawbacks of adaptation via simulation. Finally, Section $\mathrm{V}$ presents conclusions.

\section{Control Architecture}

The system dynamics can be represented as

$$
\dot{X}=F(X, \Lambda U)
$$

where $X$ is the state, $U$ is the input, and $\Lambda>0$ is the control effectiveness matrix. For control design purposes, this nonlinear plant is linearized about a trim point $\left(X_{0}, U_{0}\right)$ satisfying $F\left(X_{0}, U_{0}\right)=0$. Deviations from the trim values $X_{0}$ and $U_{0}$ will be written as lowercase letters hereafter, e.g., $X=X_{0}+x_{p}$ and $U=U_{0}+u$. Linearization about the trim point leads to the LTI system

$$
\dot{x_{p}}=A_{p} x_{p}+B_{p} \Lambda u+h\left(x_{p}, u\right)
$$

where

$$
A_{p}=\left.\frac{\partial F}{\partial X}\right|_{X_{0}, U_{0}} \quad B_{p}=\left.\frac{\partial F}{\partial U}\right|_{X_{0}, U_{0}}
$$

and $h\left(x_{p}, u\right)$ contains higher order terms. Equation 2 can be written as

$$
\dot{x}_{p}=A_{p}(\hat{p}) x_{p}+B_{1} \Lambda(\hat{p})\left(R_{s}(u)+d\right)+B_{2} \hat{r}
$$

where $A_{p}$ and $\Lambda$ are unknown matrices that depend on the uncertain parameter vector $\hat{p}, d(t)$ is an exogenous disturbance, $\hat{r}$ is the reference command, and $R_{s}(u)$ is a saturation function that enforces control saturation limits. The nominal value of $\hat{p}$, denoted as $\bar{p}$, corresponds to the case when no uncertainties or failures occur.

The state $x_{p}$ consists of angle of attack $\alpha$, sideslip angle $\beta$, aerodynamic speed $V$, roll rate $p$, pitch rate $q$, yaw rate $r$, longitude $x$, latitude $y$, altitude $z$, and the Euler angles $\psi, \theta$, and $\phi$. The control input $u$ consists of the elevators deflection $\delta_{e}$, the ailerons deflection $\delta_{a}$, the rudders deflection $\delta_{r}$, the throttle input to the left engine $\delta_{t h L}$ and the throttle input to the right engine $\delta_{t h R}$. The reference command $r$ consist of angle of attack-, sideslip-, aerodynamic speed- and roll rate-commands. These four commands, denoted hereafter as $\alpha_{\mathrm{cmd}}, \beta_{\mathrm{cmd}}, V_{\mathrm{cmd}}$ and $p_{\mathrm{cmd}}$ respectively, are generated by the pilot to attain the desired flight maneuver. Both the nominal and adaptive controllers are based on a single trim point design. Details of the procedure used to design these controllers are presented next.

\section{A. Nominal Controller}

The nominal controller consists of independent controllers for the longitudinal and the lateral/directional dynamics. Both controllers assume a multivariate LQR-PI structure having integral error states for each of the components of the reference command $\hat{r}$. Furthermore, strategies preventing the integration windup caused by input saturation are applied. A fixed control allocation matrix that correlates inputs of the same class is used to determine the ten main plant inputs. As a result, out of these ten inputs only four are independent.

\section{Longitudinal Controller}

The plant in the longitudinal axis takes the form

$$
\dot{x}_{\mathrm{lon}}=A_{\mathrm{lon}} x_{\mathrm{lon}}+B_{\mathrm{lon}} u_{\mathrm{lon}}
$$


where $A_{\text {lon }} \in \mathbb{R}^{3 \times 3}$ is the system matrix, $B_{\text {lon }} \in \mathbb{R}^{3 \times 2}$ is the input matrix, $x_{\text {lon }}=[\alpha q V]^{\top}$ is the state and $u_{\text {lon }}=\left[\delta_{e} \delta_{t h}\right]^{\top}$ is the input. To enable command tracking for angle of attack and airspeed, the integral error states

$$
\begin{aligned}
& e_{\alpha}=\int\left(\alpha-\alpha_{\mathrm{cmd}}\right) d t \\
& e_{V}=\int\left(V-V_{\mathrm{cmd}}\right) d t
\end{aligned}
$$

are added. This leads to the augmented plant

$$
\left[\begin{array}{c}
\dot{x}_{\mathrm{lon}} \\
\dot{e}_{\alpha} \\
\dot{e}_{V}
\end{array}\right]=\left[\begin{array}{c|c}
A_{\mathrm{lon}} & 0 \\
\hline H_{1} & 0
\end{array}\right]\left[\begin{array}{c}
x_{\mathrm{lon}} \\
\hline e_{\alpha} \\
e_{V}
\end{array}\right]+\left[\begin{array}{c}
B_{\mathrm{lon}} \\
0
\end{array}\right]\left[\begin{array}{c}
\delta_{e} \\
\delta_{t h}
\end{array}\right]+\left[\begin{array}{c}
0 \\
-I
\end{array}\right]\left[\begin{array}{c}
\alpha_{\mathrm{cmd}} \\
V_{\mathrm{cmd}}
\end{array}\right]
$$

An LQR-PI controller that minimizes

$$
J=\int\left(x^{T} Q x+u^{T} R u\right) d t
$$

where $Q=Q^{\top} \geq 0, R=R^{\top}>0$ are weighting matrices, is designed. This leads to

$$
\left[\begin{array}{c}
\delta_{e} \\
\delta_{t h}
\end{array}\right]=\left[\begin{array}{ll}
K_{\mathrm{lon}} & K_{e}
\end{array}\right]\left[\begin{array}{c}
x_{\mathrm{lon}} \\
e_{\alpha} \\
e_{V}
\end{array}\right]
$$

This controller must attain ample stability margins so the inclusion of the low-pass- and anti-aliasing-filters from the sensors and the delay caused by telemetry do not compromise stability.

The plant's input is given by

$$
R_{s}(u)= \begin{cases}u & \text { if } u_{\min }<u<u_{\max } \\ u_{\max } & \text { if } u \geq u_{\max } \\ u_{\min } & \text { otherwise }\end{cases}
$$

where $u$ is the controller's output, and $u_{\max }$ and $u_{\min }$ are the saturation limits of the actuator. The control deficiency caused by this saturation function is given by

$$
u_{\Delta}=R_{S}(u)-u .
$$

Details of a resetting-based anti-windup technique are presented next. The aim of anti-windup compensation is to modify the dynamics of a control loop during control saturation so that an improved transient behaviour is attained after desaturation. This practice mitigates the chance of having limit cycle oscillations and successive saturation. The anti-windup technique used prevents the occurrence of excessively large controller outputs by imposing virtual saturation limits to the integral error state used for feedback. Let $\langle e, \delta\rangle$ denote a strongly coupled pair of an integral error state $e$ and a plant input $\delta$. The anti-windup scheme proposed is governed by the saturation function

$$
R_{e}(e)= \begin{cases}e & \text { if } R_{2} \leq e \leq R_{1} \\ R_{1} & \text { if } R_{1} \leq e \\ R_{2} & \text { if } e \leq R_{2}\end{cases}
$$

where the limits $R_{1}$ and $R_{2}$ are time-varying functions assuming the smallest value of $e$ for which the plant input is equal to any of its saturation values $u_{\min }$ or $u_{\max }$. The integral error state is reset to the virtual saturation limit when $\dot{e}(t)=0$ and either $u<u_{\min }$ or $u>u_{\max }$. Analogous to Equation 111, the error deficiency caused by the anti-windup logic is

$$
e_{\Delta}=R_{e}(e, \delta)-e .
$$

The saturated value of the integral error state $R_{e}(e)$, not the integral error state itself $e$, will be used for feedback. Additional details of this technique are available in $[4,8]$. 
In the longitudinal controller case, we apply this strategy to the $\left\langle e_{\alpha}, \delta_{e}\right\rangle$ pair. Equation 10 becomes

$$
\left[\begin{array}{c}
\delta_{e} \\
\delta_{t h}
\end{array}\right]=\left[\begin{array}{ll}
K_{\mathrm{lon}} & K_{e}
\end{array}\right]\left[\begin{array}{c}
x_{\mathrm{lon}} \\
R_{e}\left(e_{\alpha}\right) \\
e_{V}
\end{array}\right]
$$

The substitution of $u$ and $e$ with $R_{s}(u)$ and $R_{e}(e, \delta)$ into Equation (8) for $u=\delta_{e}$, and of $e=e_{\alpha}$ leads to

$$
\begin{aligned}
{\left[\begin{array}{c}
\dot{x}_{\text {lon }} \\
\dot{e}_{\alpha} \\
\dot{e}_{V}
\end{array}\right] } & =\left[\begin{array}{cc}
A_{\text {lon }}+B_{\text {lon }} K_{\text {lon }} & B_{\text {lon }} K_{e} \\
H_{1} & 0
\end{array}\right]\left[\begin{array}{c}
x_{\text {lon }} \\
e_{\alpha} \\
e_{V}
\end{array}\right] x_{1}+\left[\begin{array}{c}
B_{\text {lon }} \\
0
\end{array}\right]\left[\begin{array}{c}
u_{\alpha, \Delta} \\
u_{V, \Delta}
\end{array}\right] \\
& +\left[\begin{array}{c}
B_{\text {lon }} \\
0
\end{array}\right] K_{e}\left[\begin{array}{c}
e_{\alpha, \Delta} \\
0
\end{array}\right]+\left[\begin{array}{c}
0 \\
-I
\end{array}\right]\left[\begin{array}{c}
\alpha_{\text {cmd }} \\
V_{\text {cmd }}
\end{array}\right]
\end{aligned}
$$

This Linear Time Varying (LTV) system prescribes the closed-loop longitudinal dynamics with anti-windup. The boundedness of the resulting system can be established for all initial conditions inside a bounded set. This bounded set extends to the entire state-space when the open-loop plant is stable and there are no unmodeled dynamics.

\section{Lateral/Directional Controller}

An LTI model of the corresponding plant is

$$
\dot{x}_{\text {lat }}=A_{\text {lat }} x_{\text {lat }}+B_{\text {lat }} u_{\text {lat }}
$$

where $A_{\text {lat }} \in \mathbb{R}^{3 \times 3}$ is the system matrix, $B_{\text {lat }} \in \mathbb{R}^{3 \times 2}$ is the input matrix, $x_{\text {lat }}=[\beta p r]^{\top}$ is the state, and $u_{\text {lat }}=\left[\delta_{a} \delta_{r}\right]^{\top}$ is the input. To enable satisfactory command following, integral error states for sideslip and roll rate, given by

$$
\begin{aligned}
& e_{\beta}=\int\left(\beta-\beta_{\mathrm{cmd}}\right) d t \\
& e_{p}=\int\left(p-p_{\mathrm{cmd}}\right) d t
\end{aligned}
$$

are added. The integral error in sideslip was chosen over that of the yaw rate to facilitate the generation of commands for coordinated turns with non-zero bank angles and for cross-wind landing. The augmented plant is given by

$$
\left[\begin{array}{c}
\dot{x}_{\text {lat }} \\
\dot{e}_{\beta} \\
\dot{e}_{p}
\end{array}\right]=\left[\begin{array}{c|c}
A_{\text {lat }} & 0 \\
\hline H_{2} & 0
\end{array}\right]\left[\begin{array}{c}
x_{\text {lat }} \\
\hline e_{\beta} \\
e_{p}
\end{array}\right]+\left[\begin{array}{c}
B_{\text {lat }} \\
0
\end{array}\right] u_{\text {lat }}+\left[\begin{array}{c}
0 \\
-I
\end{array}\right]\left[\begin{array}{c}
\beta_{\mathrm{cmd}} \\
p_{\mathrm{cmd}}
\end{array}\right]
$$

where $x_{2}=\left[x_{\text {lat }}^{\top} e_{\beta} e_{p}\right]^{\top}$. A LQR-PI control structure for the lateral controller is adopted. This leads to

$$
\left[\begin{array}{c}
\delta_{a} \\
\delta_{r}
\end{array}\right]=\left[\begin{array}{lll}
K_{\mathrm{lat}} & K_{e_{\beta}} & K_{e_{p}}
\end{array}\right]\left[\begin{array}{c}
x_{\mathrm{lat}} \\
e_{\beta} \\
e_{p}
\end{array}\right]
$$

As before, ample stability margins should be attained to accommodate for the low-pass filters and time delays. The anti-windup technique presented earlier is applied to the $\left\langle e_{\beta}, \delta_{r}\right\rangle$ and $\left\langle e_{p}, \delta_{a}\right\rangle$ pairs.

\section{Control Allocation}

Equations (10) and (21) along with the three realizations of the anti-windup technique mentioned above, prescribe the

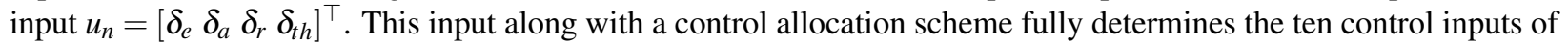
the aircraft. This relationship can be written as

$$
u_{\mathrm{nom}}=G_{\mathrm{nom}} K_{n}\left[x_{\mathrm{lon}} e_{\alpha} e_{V} x_{\mathrm{lat}} e_{\beta} e_{p}\right]^{\top},
$$

where $G_{\text {nom }} \in \mathbb{R}^{10 \times 4}$ is the control allocation matrix, and $K_{n}$ is the feedback gain. The allocation of $u_{n}$ enforced by $G$ makes the deflection of the four elevators equal, the thrust of both engines equal, the deflection of both rudders equal, and the deflection of both ailerons are equal in magnitude with opposite directions.

${ }^{\text {a }}$ The inability of a single LTI model to accurately describe the engine dynamics made the anti-windup logic for $\left\langle e_{V}, \delta_{t h}\right\rangle$ ineffective. For this reason Equation 16, only has anti-wind up for $\left\langle e_{\alpha}, \delta_{e}\right\rangle$. 


\section{B. Adaptive Controller}

The second component of the ACTS is an adaptive component that augments the nominal controller. A sketch of the resulting architecture is shown in Figure 1. The adaptive controller generates independent signals for the three main control surfaces as well as for each throttle input. This enables using the engines for attitude control. An immediate consequence of integrating the engines into the flight control system is the enlargement of the failure set where the vehicle remains controllable. The augmentation of the nominal input leads to

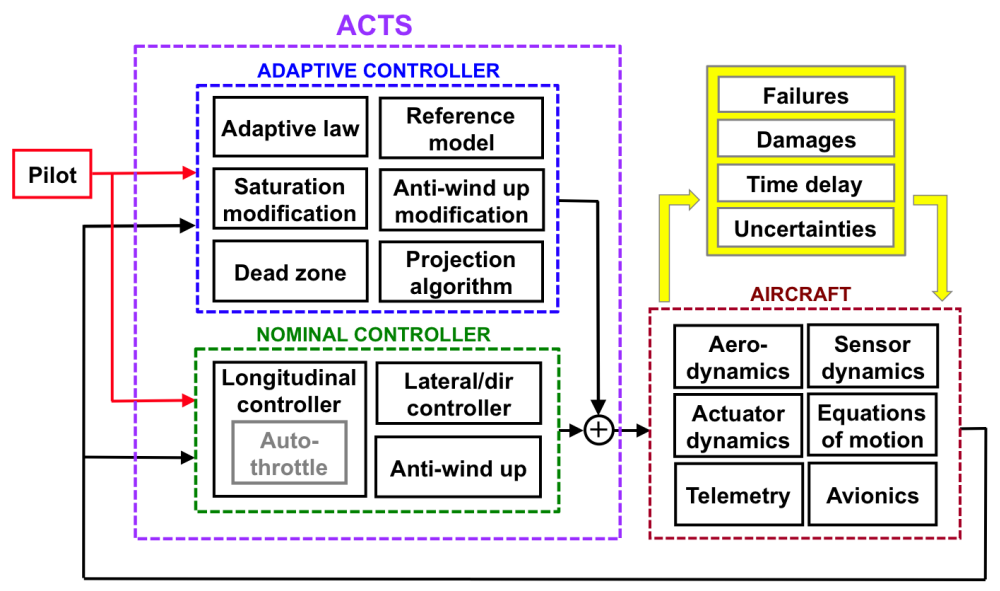

Figure 1. Control Architecture.

$$
u=u_{\mathrm{nom}}+u_{\mathrm{ada}}
$$

where $u_{\text {ada }}$ is the plant input generated by the adaptive controller. Notice that the LTI systems used for control design are accurate approximations to the aircraft dynamics as long as such dynamics are weakly coupled. However, for many of the failures and uncertainties that can occur, there will be strong coupling, e.g., when both left elevators are locked-in-place with a non-zero deflection. In such a case, the adaptive component of the controller, whose underlying dynamic model is coupled, will be active. The control gains in $u_{\text {nom }}$ will be set according to the desired system's performance under nominal flying conditions and robustness considerations for which the adaptive controller is not designed for. The control parameters in $u_{\text {ada }}$ on the other hand, will be set according to the desired levels of robustness to parametric uncertainty. Note that any nominal controller, regardless of its structure and design methodology, can be augmented with an adaptive controller.

\section{Reference Model}

The reference model is a component of the adaptive controller responsible for setting the desired closed-loop dynamics. Notice that such dynamics are the target dynamics regardless of the uncertainty/failure that may occur on the plant. The reference model assumed is prescribed by the linear closed-loop system corresponding to the nominal controller under nominal flying conditions (i.e., $\hat{p}=\bar{p}$ ). The anti-windup modifications made to the nominal controller are excluded from the reference model. This leads to the following reference model state equation:

$$
\dot{x}_{m}=\underbrace{\left[A_{p}(\bar{p})+B_{1} \Lambda(\bar{p}) G_{\mathrm{nom}} K_{n}\right]}_{A_{m}} x_{m}+B_{m} \hat{r}
$$

where $A_{m} \in \mathbb{R}^{10 \times 10}, B_{m} \in \mathbb{R}^{10 \times 3}, x_{m}=\left[\alpha \beta V p q r e_{\alpha} e_{\beta} e_{p} e_{V}\right]^{\top}$, and $\hat{r}=\left[\alpha_{\mathrm{cmd}} V_{\mathrm{cmd}} \beta_{\mathrm{cmd}} p_{\mathrm{cmd}}\right]^{\top}$. This model will be used in the design of the adaptive controller. However, this is not the same model we will use to calculate $x_{m}$ during implementation. Details on the process by which this model is derived are provided in Section III]A.

\section{Adaptive Law}

The plant to be controlled assumes the LTI representation

$$
\dot{x}_{p}=A_{p}(\hat{p}) x_{p}+B_{1} \Lambda(\hat{p})\left(R_{s}(u)+d\right)+B_{2} \hat{r}
$$


where $A_{p} \in \mathbb{R}^{10 \times 10}, B_{1} \in \mathbb{R}^{10 \times 5}, \Lambda=\operatorname{diag}\{\lambda\} \in \mathbb{R}^{5 \times 5}$ and $B_{2} \in \mathbb{R}^{10 \times 4}$. The states, inputs, and commands in 25] are

$$
\begin{aligned}
& x_{p}=\left[\begin{array}{lllll}
x_{\text {lon }}^{\top} & x_{\text {lat }}^{\top} e_{\alpha} & e_{V} & e_{\beta} & e_{p}
\end{array}\right]^{\top} \\
& u=\left[\begin{array}{lllll}
\delta_{e} & \delta_{a} & \delta_{r} & \delta_{t h_{L}} & \delta_{t h_{R}}
\end{array}\right]^{\top}
\end{aligned}
$$

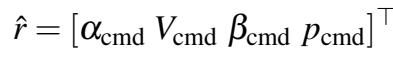

while $d \in \mathbb{R}^{5 \times 1}$ is a vector of input disturbances.

The adaptive input is given by

$$
u_{\mathrm{ada}}=G_{\mathrm{ada}} \theta^{\top} \omega=\left[\theta_{x} \theta_{d}\right]\left[\begin{array}{c}
\hat{x}_{p} \\
1
\end{array}\right]
$$

where $G_{\text {ada }} \in \mathbb{R}^{10 \times 5}$ is a control allocation matrix, $\theta_{x} \in \mathbb{R}^{5 \times 10}$ and $\theta_{d} \in \mathbb{R}^{5 \times 1}$ are adaptive parameters, and

$$
\hat{x}_{p}=\left[x_{\text {lon }}^{\top} x_{\text {lat }}^{\top} f\left(e_{\alpha}\right) e_{V} e_{\beta} e_{p}\right]^{\top}
$$

is the state being fed back. $G_{\text {ada }}$ makes the deflection of the four elevators equal, the deflection of both rudders equal, and the deflection of both ailerons equal in magnitude with opposite directions. Adaptive laws without the resettingbased anti-windup modification make $f$ equal to its argument so $\hat{x}_{p}=x_{p}$. The implementation of this anti-wind up scheme for the pair $\left\langle e_{\alpha}, \delta_{e}\right\rangle$ uses $f\left(e_{\alpha}\right)=R_{e}\left(e_{\alpha}, \delta_{e}\right)$. The adaptive gains are given by

$$
\begin{aligned}
\dot{\theta} & =\operatorname{Proj}\left\{-\Gamma_{1} \omega e_{u}^{\top} P B_{1} \operatorname{sign}(\Lambda) \Gamma_{2}, \theta_{\max }\right\} \\
\dot{\hat{\lambda}} & =-\Gamma_{\lambda} \operatorname{diag}(\kappa) B_{1}^{\top} P e_{u} \\
\dot{e}_{\Delta} & =A_{m} e_{\Delta}-B_{1} \operatorname{diag}(\hat{\lambda}) \kappa \\
\kappa & =u_{\Delta}+\left(K_{e_{\alpha}}^{\top}+\theta_{e_{\alpha}}^{\top}\right) e_{\alpha, \Delta}
\end{aligned}
$$

where $\operatorname{Proj}\{\cdot\}$ is the projection operator [7], $e_{u}=e-e_{\Delta}, P=P^{\top}>0$ satisfies $A_{m}^{\top} P+P A_{m}=-Q$ for a fixed $Q=Q^{\top}>0$, $e=x_{p}-x_{m}$, and $u_{\Delta}$ is the multivariable version of the input deficiency in Equation $(12)$. While $e_{\Delta}$ is the error caused by saturation in the control inputs and in the integral error state $e_{\alpha}, e_{u}$ is the error caused by parametric uncertainties. $\kappa$, which along with $f_{1}$ constitutes the anti-windup modification, depends on the column vectors of $K_{n}$ and $\theta$ corresponding to $R_{e}\left(e_{\alpha}, \delta_{e}\right)$ and $f_{1}\left(e_{\alpha}\right)$ respectively. The variables $Q>0, \Gamma_{1} \in \mathbb{R}^{11 \times 11}>0, \Gamma_{2} \in \mathbb{R}^{5 \times 5}>0$, $\theta_{\max } \in \mathbb{R}^{11 \times 11}>0$ and $\Gamma_{\lambda} \in \mathbb{R}^{5 \times 5}>0$ are design parameters that determine the rate of adaptation ${ }^{b}$ In practice, the adaptive rates $\Gamma_{1}$ and $\Gamma_{\lambda}$ are made zero when the effects of nonlinearities and time delays are prevalent. As a result, these adaptive rates become piecewise constant functions that may depend on the state of the aircraft $x$, the pilot command $\hat{r}$, the control input $u$, and the error $e$. The dead zones as well as $\theta_{\max }$ determine the range of adaptation.

Note that the integral error states depend on the command $\hat{r}$, but the error $e$ driving adaptation does not. The adaptive controller achieves command tracking by making $x_{p}$ follow $x_{m}$ via the adaptive law, and by having a stable reference model (i.e., $x_{m}(\infty) \rightarrow 0$ implies the convergence of the reference model states to the pilot commands).

This adaptive law makes the plant track the dynamics of the reference model, accommodates for control saturation, and mitigates integral windup in $e_{\alpha}$. This architecture requires that all the components of $x_{p}, e_{\alpha, \Delta}$ and $u_{\alpha, \Delta}$ be accessible. Notice that anti-windup logic is now extended to $u$ as a whole, and not to $u_{\text {nom }}$ and $u_{\text {ada }}$ independently. However for the $\left\langle e_{V}, \delta_{t h}\right\rangle,\left\langle e_{\beta}, \delta_{r}\right\rangle$ and $\left\langle e_{p}, \delta_{a}\right\rangle$ pairs, anti-windup is only applied to $u_{\text {nom. }}$. The strong coupling between $\beta$ and $p$ makes the adaptive anti-wind up scheme for $\left\langle e_{\beta}, \delta_{a}\right\rangle$ and $\left\langle e_{p}, \delta_{r}\right\rangle$ ineffective. As before, inaccuracies in the LTI representation of the engine dynamics yield the same outcome for $\left\langle e_{V}, \delta_{t h}\right\rangle$. The Lyapunov stability analysis in reference [8] demonstrates that for a bounded set of commands, $\theta, x$ and $e$ are semi-globally bounded. This result holds under the assumption that the disturbances, time-delays and unmodeled dynamics are not present and that both the plant and the reference model are LTI.

\section{Control Implementation}

This section presents a few aspects of the controller's implementation that depart from the LTI framework used for control design.

\footnotetext{
${ }^{\mathrm{b}}$ Note that $\Gamma_{1}^{-1}$ and $\Gamma_{2}^{-1}$ are analogous to the $Q$ and $R$ of an LQR setting. $\Gamma_{1}$ determines the rate of adaptation as a function of the state $\omega$, while $\Gamma_{2}$ determines the rate of adaptation as a function of the control inputs. Rates of adaptation for states and inputs are inversely proportional to the $Q$ and $R$ penalty matrices of $\mathrm{LQR}$
} 


\section{A. Reference Model}

Due to nonlinearities, the dynamics set by the linear reference model in Equation (24) may differ considerably from those of the actual aircraft. These nonlinearities will undesirably trigger adaptation. Since the primary objective of adaptive control is to compensate for parametric uncertainties -not for nonlinear dynamics- this situation may seriously compromise the stability and performance characteristics of the controller. In this section we examine alternatives that expand the region of the state space where the reference model accurately describes the closed-loop aircraft dynamics corresponding to the nominal controller.

One choice of the reference model is to use a full nonlinear model. Even though this will directly account for the main nonlinearities and eliminate adapting to them, the computational requirements associated with it may be exceedingly high. This complexity results from having to perform a high fidelity simulation in real time as well as to having to verify and validate the software and the hardware.

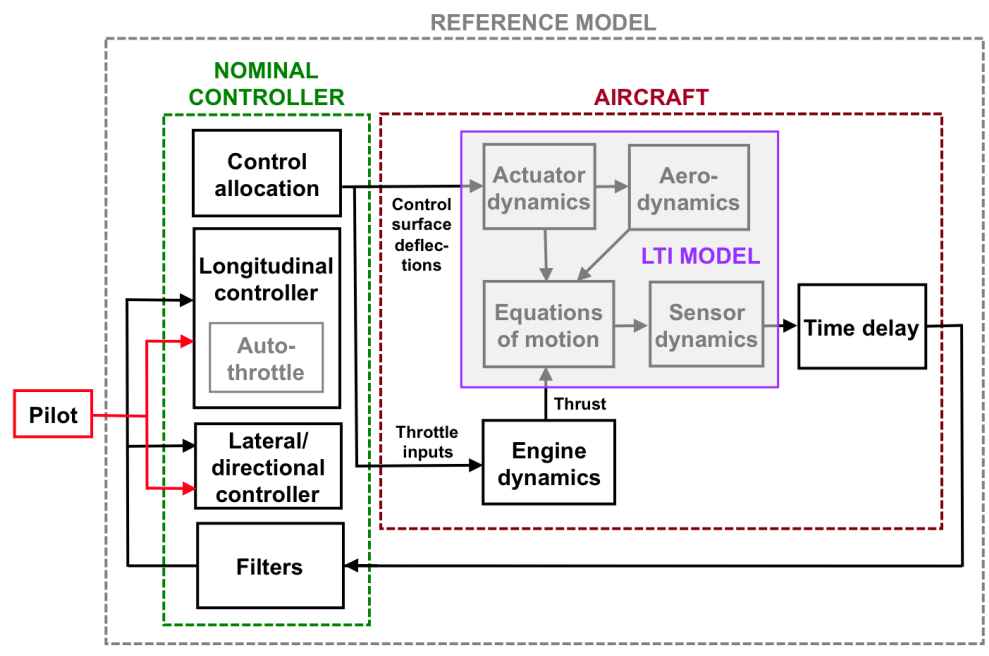

Figure 2. Reference model.

The search for an accurate yet simple reference model led us to a system with the following features: (i) the underlying structure of the plant is LTI (see Section IV $\mathrm{D}$ for an exception to this statement), (ii) there is an engine model to accurately describe the nonlinear dependency of the thrust on the engine's RPMs, (iii) there is uplink time delay between the controller and the plant capturing the effects of telemetry and signal processing, (iv) there is a down link time delay due to the sensor dynamics, (v) there is a bank of low pass filters that mitigate sensor noise, and (vi) there are anti-aliasing filters and command rate limiters as in the actual GTM. The states and inputs of the reference model are $x=\left[\alpha \beta V p q r x y z \psi \theta \phi e_{\alpha} e_{V} e_{\beta} e_{p}\right]^{\top}$ and $u=\left[\delta_{e} \delta_{a} \delta_{r} \delta_{t h}\right]^{\top}$. Note that $x_{m}$ in Equation 24] is a subset of $x$. Figure 2 shows the main components of this reference model.

\section{B. Adaptive Rate}

In the LTI framework supporting the theory, asymptotic tracking and stability are guaranteed for any adaptive rates satisfying $\Gamma_{1}>0, \Gamma_{2}>0$ and $\Gamma_{\lambda}>0$. In such a setting, the larger the adaptive rate the faster the adaptation and the better the performance. This is not the case when there are nonlinearites and time delays in the plant. While excessively small adaptive rates diminish the performance advantages resulting from adaptation by practically turning the adaptive controller off, excessively large ones induce high frequency oscillations that can lead to instability. The challenge from the control designer perspective is to balance these two behaviours. Furthermore, noise, hysteresis and actuator's dead zones have the potential of degrading the closed-loop system's performance considerably. A dead zone for the adaptive controller, where $\Gamma_{1}$ and $\Gamma_{\lambda}$ are piecewise constant functions of the tracking error $e_{u}$, the aircraft's state $x_{p}$, and of the total input $u$, can be used to counteract these anomalies. 


\section{Simulation Studies}

In this section we showcase some of the advantages and disadvantages of adaptation via simulation. For this we use a high-fidelity model of the aircraft, a flight-validated nominal controller [3] (which prescribes the reference model dynamics in all cases) and several adaptive controllers only differing in the value of their tunable control parameters.

\section{A. Trade-Offs}

Figure 3 shows the closed-loop response for the nominal and adaptive controllers when the effectiveness of the elevators is reduced to $50 \%$ and a severe degradation in pitch stiffness $C_{m \alpha}$ and roll damping $C_{l p}$ occur. Note that the nominal controller is unable to stabilize the pitch dynamics. The adaptive controller on the other hand, not only stabilizes these dynamics but also improves roll command tracking significantly. This is a situation where adaptation yields a significant improvement in flight safety. Unfortunately, this adaptive controller is overly aggressive for other types of
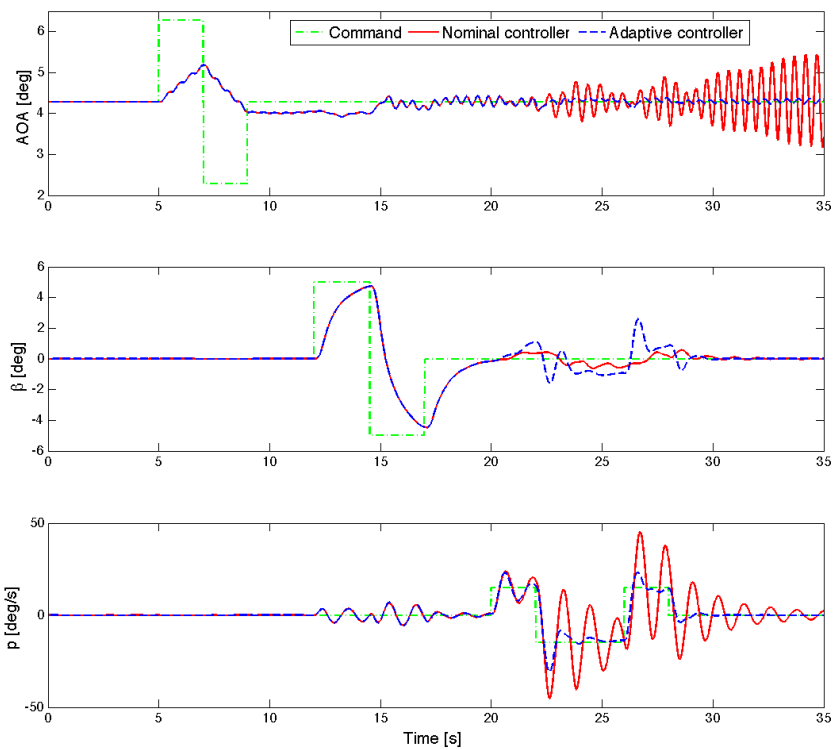

Figure 3. Closed-loop response corresponding to the nominal and adaptive controllers.

uncertainties. Figure 4 shows the closed-loop response for the same controllers when there is an additional uplink time delay of $60 \mathrm{~ms}$. While the non-adaptive controller achieves command tracking with minimal residual oscillations, the performance degradation caused by adaptation makes the controller unacceptable. There are other uncertainty/failure realizations for which this adaptive controller triggers instability. In these situations it is adaptation itself that compromises safety. These cases highlight the importance of prescribing adaptive rates that are large enough to compensate for parametric uncertainty without magnifying the adverse effects of nonlinearities and time delays.

\section{B. Unrealizable Reference Model Dynamics}

It is important to determine if the reference model prescribes a dynamics that the actual aircraft is able to realize (even in the case where the vehicle remains controllable after a failure). If physical limitations prevent the aircraft from attaining the dynamics set by the reference model -say due to failure, damage or uncertainty- the controller itself, and thereby the plant, may become unstable. This instability, which is triggered by the the controller, can be avoided if a more suitable reference model is used. Figure 5 shows the closed-loop response corresponding to an adaptive controller having a flawed reference model. No uncertainty or failure occurs. The source of the discrepancy between the plant and the reference model is a $2 \mathrm{~Hz}$ difference in the the value of the bandwidth of a low pass filter. In this simulation, periodic wave trains for $\alpha_{\mathrm{cmd}}$ and $p_{\mathrm{cmd}}$ are used to aggravate the effects of the mismatch. Note that as time goes on, the ability of the controller to track the reference model worsens progressively. This progression, which is caused by the growth of the integral error states in $e$, will eventually lead to instability. 

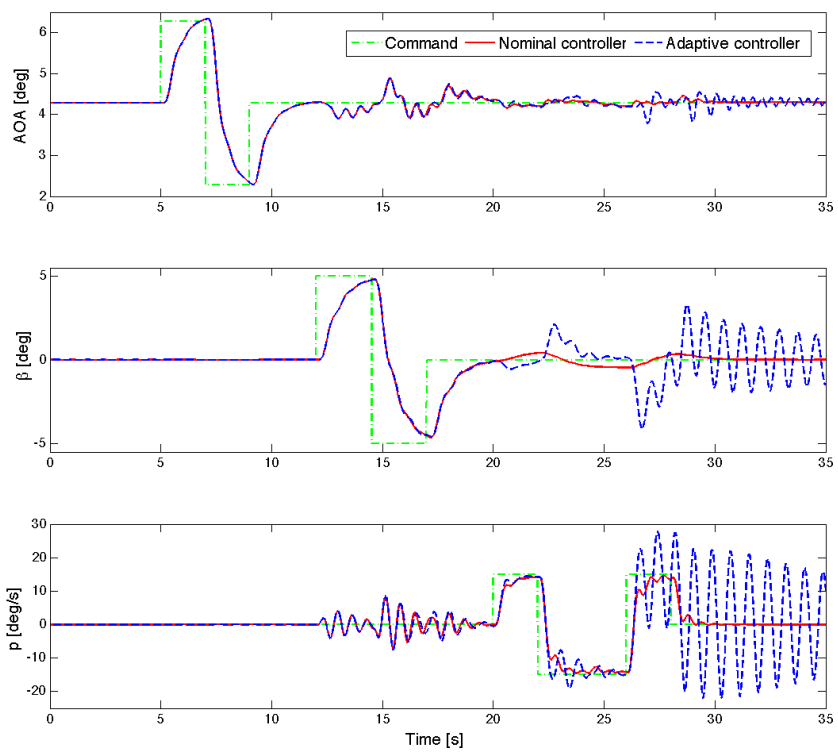

Figure 4. Closed-loop response corresponding to the nominal and adaptive controllers.
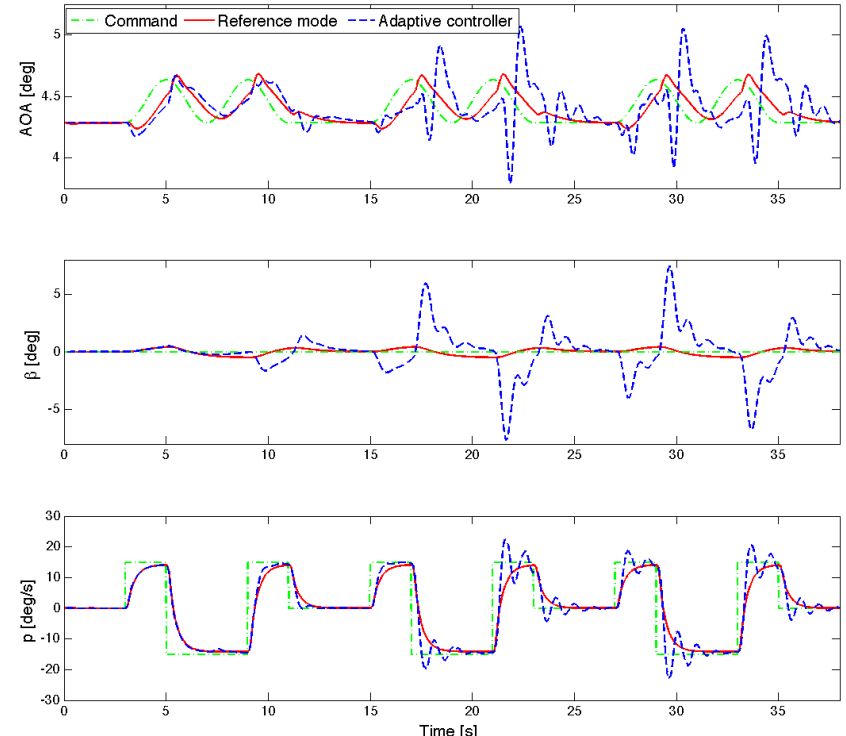

Figure 5. Effects of unrealizable model reference dynamics. 
Note that the adaptive controller will react to any mismatch between the dynamics of the aircraft and the dynamics of the reference model regardless of its origin. While reactions to parametric uncertainties entering the equations of motion according to the structure of the matching conditions are desirable, all others are not. In order to prevent these other mismatches from triggering adaptation, the adaptive rate should be made zero. The switching between zero and non-zero adaptive rates can be attained by prescribing adaptive dead zones. These zones may depend on the state $x$, the control input $u$, the command $\hat{r}$, and the error $e$. The key aspect of designing these dead zones is determining when to apply them. This determination requires knowing the region in the flight envelope where the reference model represents the aircraft dynamics accurately. All efforts aiming at evaluating or expanding this region are beneficial.

\section{Actuator Anomalies: Surface Dead Band}

Figure 6 shows the effects of control surface dead band on the same adaptive controller used in Figure 3 for the same uncertainty/failure realization. Even though asymptotic tracking is ultimately achieved, the significant degradation in the transient response makes the controller unacceptable. It is worth highlighting that the effects of this actuator anomaly on the nominal controller are barely noticeable. In practice, the nonlinear effects of anomalies like this one (e.g., hysteresis) are a function of the aircraft's state, and as such, strategies based on their off-line inversion may not be effective. Since the aircraft is uncontrollable within the surface dead zone, the adaptive controller fails to drive
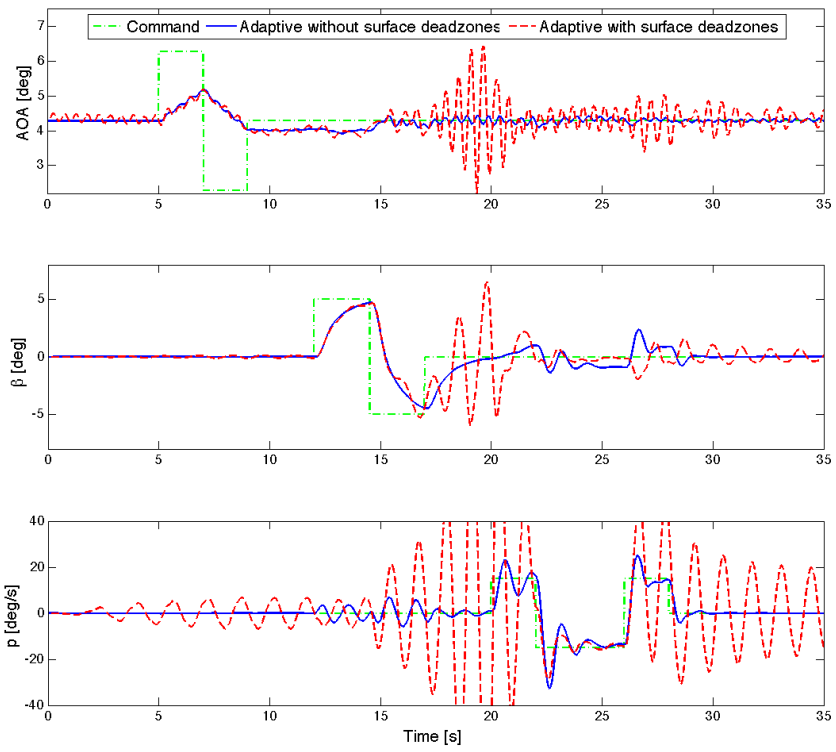

Figure 6. Effects of dead zones in control surfaces.

the error to zero. Small residues in the integral error states of this error will build up over time. When these terms are sufficiently large, they will make the input $u$ large enough to leave the dead zone. Once the inputs are out of the dead zone and the control surfaces are effective again, the controller will drive the inputs back into the dead zone. This chattering cycle will keep repeating unless a controller dead band is applied. These dead bands, however, reduce the ability of the adaptive controller to compensate for parametric uncertainty. Better strategies can be applied if measurements of the control surface deflections are available. By removing the effects of the actuator anomalies from the error used for adaptation, i.e., $u_{\Delta}=u-u_{\text {measured }}$ in Equation (27), the adaptive controller will only react to the parametric uncertainties it was designed for.

\section{Knowing the Sign of $\Lambda$}

There are physical effects for which a single LTI system cannot capture the aircraft dynamics accurately regardless to the proximity of the state to the equilibrium point used for linearization. For instance, the loss in altitude caused by either a positive or a negative aileron deflection cannot be modelled with a fixed input matrix $B$. A fixed $B$ matrix leads to a situation where rolling to one side will decrease altitude while rolling to the other one will increase it. This 
physics of this phenomenon are better modelled as $B|u|$. Even though this is a second order effect in the coupling between the longitudinal and lateral/directional dynamics, it has the potential to make the reference model dynamics unrealizable and therefore, to drive the system unstable.

Note that from the perspective of the adaptive controller this is equivalent to not knowing the signs of the entries of $\Lambda$ in Equation (29). In order to prevent these difficulties, the sign of some of the components of the $B_{1}$ matrix in Equations (29)-(31) and in the reference model are switched according to the instantaneous value of the input. Figure
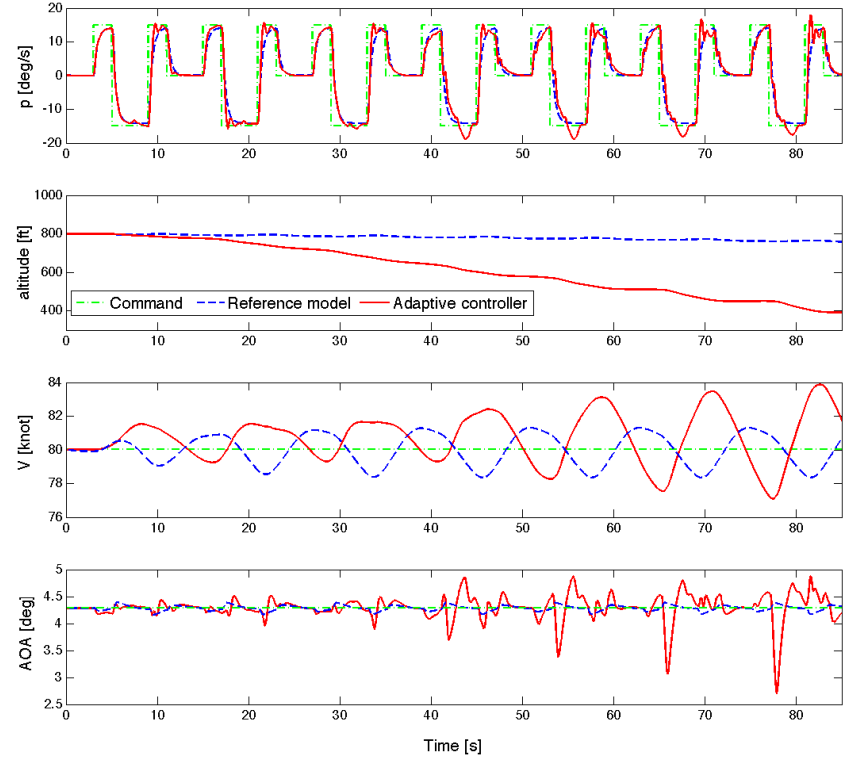

Figure 7. Growing offset between LTI reference model and nonlinear plant.

7 illustrates the consequences of using a fixed input matrix $B$ when a periodic train of roll rate commands is input. The same type of "build-up" behavior observed in Figure 5 occurs. Note that the peaks in the angle of attack and progressively larger variations in velocity caused by the loss in altitude are absent from the dynamics of the reference model. In its attempt to make the falling aircraft respond as if it were a wings-level flight, adaptation ends up creating a large phase difference between the velocities of the aircraft and of the reference model. This phenomenon makes the integral error state of the controller $e_{V}$ to grow unbounded.

\section{E. Counter-acting Actuation}

The control inputs generated by the adaptive controller are independent signals. If some of these inputs correspond to actuators with similar functions (e.g., there is an input for each of the two rudder segments), the adaptive controller is performing both, control design and control allocation. This independence may lead to a configurations where the adaptive inputs counteract each other. For instance, a situation may arise where the upper and lower rudders deflect in opposite directions so their overall effect on the yaw dynamics is nil. Even though this configuration may be able to achieve asymptotic tracking, the incidental increase in drag and fuel consumption makes the controller unacceptable. The simplest way to avoid this outcome is to restrict the adaptive controller to use non-redundant inputs. The resulting controller outputs will then be allocated using traditional control allocation methods. This practice, however, eliminates degrees of freedom the adaptive controller could take advantage of (e.g., the differential use of elevators may be able to compensate for aileron failures).

\section{Conclusions}

This paper develops and evaluates an adaptive controller for an unmanned air vehicle. The aim of the control design and control implementation procedures, which encompass both theoretical and practical considerations, is to develop a controller suitable for flight. While an increase in the rate of adaptation can improve robustness to aerodynamic 
uncertainties, it commonly yields a reduction in time delay margin. Furthermore, adequate rates of adaptation for some uncertainty realizations may be overly-aggressive for others. Excessively large adaptive rates along with nonlineatities and time delays have the potential of driving the controller, and thereby the aircraft, unstable. This setting defines a trade-off from which the the adaptation rates should be prescribed. Failure to do this properly may lead to controllers that compromise safety by adapting either too fast or too slow. The determination of the range of adaptation, which is the region of the flight envelope where adaptation occurs, plays a critical role when trying to fend off the effects of nonlinearities and time delays. Practical aspects of developing a flight control system, such as the need for accurately modeling the aircraft dynamics, the performance degradation caused by actuator anomalies, and the consequences of prescribing overly-demanding reference models are illustrated via simulation.

\section{References}

${ }^{1}$ Karl J. Astrom and Bjorn Wittenmark. Adaptive Control. Addison-Wesley, Reading, MA, 2nd edition, 1994.

${ }^{2}$ L. G. Crespo, S. P. Kenny, and D. P. Giesy. A computational framework to control verification and robustness analysis. In NASA TP 2010-216189, pages 1-38, NASA Langley Research Center, Hampton, VA, 2010.

${ }^{3}$ L. G. Crespo, M. Matsutani, and A. Annaswamy. Verification and tuning of an adaptive controller for an unmaned air vehicle. In AIAA Guidance Navigation and Control Conference, Toronto, Canada, August 2010.

${ }^{4}$ Luis G. Crespo, M. Matsutani, and Anuradha M. Annaswamy. Design and verification of an adaptive controller for the generic transport model. In Proceedings of AIAA Guidance, Navigation, and Control Conference and Exhibit, Chicago, IL, USA, 2009. AIAA $2009-5618$.

${ }^{5}$ Petros A. Ioannou and Jing Sun. Robust Adaptive Control. Prentice Hall, Upper Saddle River, NJ, 1996.

${ }^{6}$ Y. D. Landau. Adaptive Control: The model reference approach. Marcel Dekker, New York: NY, 1979.

${ }^{7}$ Eugene Lavretsky. The projection operator. Personal Notes, 2006.

${ }^{8}$ M. Matsutani, Luis G. Crespo, and Anuradha M. Annaswamy. Application of a novel adaptive reset controller to the GTM. In Proceedings of AIAA Guidance, Navigation, and Control Conference and Exhibit, Toronto, Canada, 2010.

${ }^{9}$ Kumpati S. Narendra and Anuradha M. Annaswamy. Stable Adaptive Systems. Prentice Hall, Englewoods Cliffs, NJ, 1989.

${ }^{10}$ Shankar S. Sastry and Marc Bodson. Adaptive Control: Stability, Convergence, and Robustness. Prentice Hall, Englewood Cliffs, NJ, 1989. 